\title{
Rogue waves in the Davey-Stewartson equation
}

\author{
Yasuhiro Ohta ${ }^{1 *}$ and Jianke Yang ${ }^{2 \dagger}$ \\ ${ }^{1}$ Department of Mathematics, Kobe University, Rokko, Kobe 657-8501, Japan \\ ${ }^{2}$ Department of Mathematics and Statistics, University of Vermont, Burlington, VT 05401, U.S.A
}

\begin{abstract}
General rogue waves in the Davey-Stewartson-I equation are derived by the bilinear method. It is shown that the simplest (fundamental) rogue waves are line rogue waves which arise from the constant background with a line profile and then disappear into the constant background again. It is also shown that multi-rogue waves describe the interaction of several fundamental rogue waves. These multi-rogue waves also arise from the constant background and then decay back to it, but in the intermediate times, interesting curvy wave patterns appear. However, higher-order rogue waves are found to show more interesting features. Specifically, only part of the wave structure in the higher-order rogue waves rises from the constant background and then retreats back to it, and this transient wave exhibits novel patterns such as parabolas. But the other part of the wave structure comes from the far distance as a localized lump, which decelerates to the near field and interacts with the transient rogue wave, and is then reflected back and accelerates to the large distance again. These rogue-wave solutions have interesting implications for two-dimensional surface water waves in the ocean.
\end{abstract}

\section{INTRODUCTION}

Rogue waves are large and spontaneous ocean surface waves that occur in the sea and are a threat even to large ships and ocean liners [1]. Recently, an optical analogue of rogue waves - optical rogue waves, was also observed in optical fibres [2, 3. A growing consensus is that both oceanic and optical rogue waves appear as a result of modulation instability of monochromatic nonlinear waves. Mathematically, the first and simplest rogue-wave solution was reported in the nonlinear Schrödinger (NLS) equation by Peregrine [4]. This solution approaches a non-zero constant background as time goes to $\pm \infty$ but develops a localized hump with peak amplitude three times the constant background in the intermediate times. Recently, higher-order rogue waves in the NLS equation were reported in many articles [512. It was shown that these higher-order waves could reach higher peak amplitudes or exhibit multiple intensity peaks at different spatial locations and times. In addition to the NLS equation, rogue waves in some other wave equations (such as the Hirota equation) have also been explored [13. Rogue waves are intimately related to homoclinic solutions which approach a constant background as time goes to $\pm \infty$ but develop spatially-periodic wave patterns in the intermediate times [14 17]. Indeed, rogue waves can be obtained from homoclinic solutions when the spatial period of homoclinic solutions goes to infinity 8, 14, 15, 18.

Rogue waves which have been studied so far are mostly one-dimensional. But ocean surface waves are always two-dimensional. Thus a natural question is to investigate rogue waves in two-dimensional model equations. It is well known that the evolution of a two-dimensional

\footnotetext{
*Email: ohta@math.kobe-u.ac.jp

†Email: jyang@math.uvm.edu
}

wave-packet on water of finite depth is governed by the Benney-Roskes-Davey-Stewartson equation [19 21]. This equation is often just called the Davey-Stewartson (DS) equation in the literature. The DS equation is divided into two types, DSI and DSII equations, depending on the signs of its coefficients. The simplest (one-mode) homoclinic solution to the DS equation was derived in 22. Taking the spatial period of this homoclinic solution to go to infinity, the simplest (fundamental) rogue-wave solution was also obtained there. But more general rogue waves in the DS equation are still unknown.

In this paper, general rogue waves in the DS-I equation are derived. These solutions are obtained by the bilinear method and expressed in terms of determinants. It is shown that the simplest (fundamental) rogue waves are line rogue waves which arise from the constant background with a line profile and then disappear into the constant background again (this simplest rogue wave agrees with that reported in [22]). It is also shown that non-fundamental rogue waves contain different types such as the multi-rogue waves and higher-order rogue waves. The multi-rogue waves describe the interaction of several fundamental rogue waves. These multi-rogue waves arise from the constant background and then decay back to it, but in the intermediate times, interesting curvy wave patterns appear. Higher-order rogue waves, on the other hand, exhibit certain features which are very novel. Specifically, only parts of the wave structures in these higher-order rogue waves rise from the constant background and then retreat back to it, exhibiting unusual transient wave patterns (such as parabola shapes) in the intermediate times. But the other parts of the waves come from the far distance as localized lumps, which interact with the transient rogue waves in the near field and then are reflected back to the large distance again. Since the DS equation is a well known mathematical model for two-dimensional surface water waves, these general rogue-wave solutions can have interesting implications for the study of rogue waves in the ocean. 


\section{RATIONAL SOLUTIONS IN THE DAVEY-STEWARTSON-I EQUATION}

The Davey-Stewartson-I (DSI) equation is given by

$$
\begin{aligned}
& i A_{t}=A_{x x}+A_{y y}+\left(\epsilon|A|^{2}-2 Q\right) A, \\
& Q_{x x}-Q_{y y}=\epsilon\left(|A|^{2}\right)_{x x},
\end{aligned}
$$

where $\epsilon=1$ or -1 . It is noted that under the variable transformation $Q \rightarrow Q+\epsilon|A|^{2}, x \leftrightarrow y$ and $\epsilon \rightarrow-\epsilon$, this equation is invariant, thus we can fix the sign of $\epsilon$ without loss of generality. However the transformation $Q \rightarrow Q+\epsilon|A|^{2}$ changes the boundary condition of $Q$ in general, thus we keep $\epsilon$ in our analysis. Equation 2.1) is transformed into the bilinear form,

$$
\begin{aligned}
& \left(D_{x}^{2}+D_{y}^{2}-i D_{t}\right) g \cdot f=0, \\
& \left(D_{x}^{2}-D_{y}^{2}\right) f \cdot f=2 \epsilon\left(f^{2}-|g|^{2}\right),
\end{aligned}
$$

through the variable transformation,

$$
A=\sqrt{2} \frac{g}{f}, \quad Q=\epsilon-(2 \log f)_{x x},
$$

where $f$ is a real variable and $g$ is a complex one.

Rogue waves are rational solutions (under certain parameter restrictions). Thus we first present the general rational solutions to the DSI equation in the following theorem. The proof of this theorem is given in the appendix.

Theorem 1 The DSI equation (2.1) has rational solutions 2.3. with $f$ and $g$ given by $N \times N$ determinants

$$
f=\tau_{0}, \quad g=\tau_{1},
$$

where $\tau_{n}=\operatorname{det}_{1 \leq i, j \leq N}\left(m_{i j}^{(n)}\right)$, and the matrix elements are given by either

(a)

$$
\begin{aligned}
& m_{i j}^{(n)}= \sum_{k=0}^{n_{i}} c_{i k}\left(p_{i} \partial_{p_{i}}+\xi_{i}^{\prime}+n\right)^{n_{i}-k} \\
& \sum_{l=0}^{n_{j}} \bar{c}_{j l}\left(\bar{p}_{j} \partial_{\bar{p}_{j}}+\bar{\xi}_{j}^{\prime}-n\right)^{n_{j}-l} \frac{1}{p_{i}+\bar{p}_{j}}, \\
& \xi_{i}^{\prime}=\frac{p_{i}-\epsilon p_{i}^{-1}}{2} x+\frac{p_{i}+\epsilon p_{i}^{-1}}{2} y+\frac{p_{i}^{2}+p_{i}^{-2}}{\sqrt{-1}} t,
\end{aligned}
$$

or

(b)

$$
\begin{gathered}
m_{i j}^{(n)}=\sum_{\nu=0}^{n_{i}+n_{j}}\left(\frac{-1}{p_{i}+\bar{p}_{j}}\right)^{\nu+1}\left(\partial_{x}+\partial_{y}\right)^{\nu} P_{i}^{(n)} \overline{P_{j}^{(-n)}}, \\
P_{i}^{(n)}=\sum_{k=0}^{n_{i}} \hat{c}_{i k} S_{n_{i}-k}\left(\boldsymbol{\xi}^{(n)}\left(p_{i}\right)\right)
\end{gathered}
$$

where $S_{n}(\boldsymbol{x})$ is the elementary Schur polynomial defined via the generating function

$$
\sum_{n=0}^{\infty} S_{n}(\boldsymbol{x}) \lambda^{n}=\exp \left(\sum_{k=1}^{\infty} x_{k} \lambda^{k}\right)
$$

for $\boldsymbol{x}=\left(x_{1}, x_{2}, \cdots\right)$,

$$
\boldsymbol{\xi}^{(n)}(p)=\left[\xi_{1}(p)+n, \xi_{2}(p), \cdots, \xi_{k}(p)+\delta_{k 1} n, \cdots\right],
$$

$\delta_{i j}$ is the Kronecker delta notation (which is equal to 1 when $i=j$ and zero otherwise), and

$$
\begin{aligned}
\xi_{k}(p)=\frac{1}{k !}[ & \frac{p+\epsilon(-1)^{k} / p}{2} x+\frac{p-\epsilon(-1)^{k} / p}{2} y \\
& \left.+\frac{2^{k} p^{2}-(-2)^{k} / p^{2}}{2 \sqrt{-1}} t\right] .
\end{aligned}
$$

In (a) and (b), $p_{i}, c_{i k}$ and $\hat{c}_{i k}$ are arbitrary complex constants, and $n_{i}$ is an arbitrary positive integer. These two expressions in (a) and (b) would yield identical solutions if the constants $c_{i k}$ and $\hat{c}_{i k}$ in them are related by

$$
\hat{c}_{i k}=c_{i k}\left(n_{i}-k\right) !, \quad \hat{d}_{j l}=d_{j l}\left(n_{j}-l\right) ! .
$$

Thus in the later text we use the expression in (a). By a scaling of $f$ and $g$, we can normalize $c_{i 0}=1$ without loss of generality, thus hereafter we set $c_{i 0}=1$. We will also call the above solution as the $N$-rational solution of order $\left(n_{1}, n_{2}, \cdots, n_{N}\right)$. We comment that a more explicit expression for $m_{i j}^{(n)}$ similar to (2.6) in [12] can also be obtained, but since that expression is a bit complicated, we omit it in this paper.

The simplest rational solution, namely 1-rational solution of 1 st order, is given by taking $N=1$ and $n_{1}=1$,

$$
\begin{aligned}
& f=\sum_{k=0}^{1} c_{1 k}\left(p_{1} \partial_{p_{1}}+\xi_{1}^{\prime}\right)^{1-k} \sum_{l=0}^{1} \bar{c}_{1 l}\left(\bar{p}_{1} \partial_{\bar{p}_{1}}+\bar{\xi}_{1}^{\prime}\right)^{1-l} \frac{1}{p_{1}+\bar{p}_{1}} \\
& =\left(p_{1} \partial_{p_{1}}+\xi_{1}^{\prime}+c_{11}\right)\left(\bar{p}_{1} \partial_{\bar{p}_{1}}+\bar{\xi}_{1}^{\prime}+\bar{c}_{11}\right) \frac{1}{p_{1}+\bar{p}_{1}} \\
& =\frac{1}{p_{1}+\bar{p}_{1}}\left[\left(\xi_{1}^{\prime}+c_{11}-\frac{p_{1}}{p_{1}+\bar{p}_{1}}\right)\left(\bar{\xi}_{1}^{\prime}+\bar{c}_{11}-\frac{\bar{p}_{1}}{p_{1}+\bar{p}_{1}}\right)\right. \\
& \left.+\frac{p_{1} \bar{p}_{1}}{\left(p_{1}+\bar{p}_{1}\right)^{2}}\right] \\
& g=\sum_{k=0}^{1} c_{1 k}\left(p_{1} \partial_{p_{1}}+\xi_{1}^{\prime}+1\right)^{1-k} \\
& \sum_{l=0}^{1} \bar{c}_{1 l}\left(\bar{p}_{1} \partial_{\bar{p}_{1}}+\bar{\xi}_{1}^{\prime}-1\right)^{1-l} \frac{1}{p_{1}+\bar{p}_{1}} \\
& =\left(p_{1} \partial_{p_{1}}+\xi_{1}^{\prime}+1+c_{11}\right)\left(\bar{p}_{1} \partial_{\bar{p}_{1}}+\bar{\xi}_{1}^{\prime}-1+\bar{c}_{11}\right) \frac{1}{p_{1}+\bar{p}_{1}} \\
& =\frac{1}{p_{1}+\bar{p}_{1}}\left[\left(\xi_{1}^{\prime}+1+c_{11}-\frac{p_{1}}{p_{1}+\bar{p}_{1}}\right) \times\right. \\
& \left.\left(\bar{\xi}_{1}^{\prime}-1+\bar{c}_{11}-\frac{\bar{p}_{1}}{p_{1}+\bar{p}_{1}}\right)+\frac{p_{1} \bar{p}_{1}}{\left(p_{1}+\bar{p}_{1}\right)^{2}}\right],
\end{aligned}
$$


where

$$
\xi_{1}^{\prime}=\frac{p_{1}-\epsilon p_{1}^{-1}}{2} x+\frac{p_{1}+\epsilon p_{1}^{-1}}{2} y+\frac{p_{1}^{2}+p_{1}^{-2}}{i} t,
$$

and $p_{1}, c_{11}$ are arbitrary complex constants. This solution can be rewritten as

$f=\frac{1}{p_{1}+\bar{p}_{1}}(\xi \bar{\xi}+\Delta), \quad g=\frac{1}{p_{1}+\bar{p}_{1}}[(\xi+1)(\bar{\xi}-1)+\Delta]$,

where

$$
\xi=a x+b y+\omega t+\theta, \quad \Delta=p_{1} \bar{p}_{1} /\left(p_{1}+\bar{p}_{1}\right)^{2},
$$

and

$$
\begin{gathered}
a=\left(p_{1}-\epsilon p_{1}^{-1}\right) / 2, \quad b=\left(p_{1}+\epsilon p_{1}^{-1}\right) / 2, \\
\omega=\left(p_{1}^{2}+p_{1}^{-2}\right) / i, \quad \theta=c_{11}-p_{1} /\left(p_{1}+\bar{p}_{1}\right) .
\end{gathered}
$$

If we separate the real and imaginary parts of $a, b, \omega$ and $\theta$ as

$a=a_{1}+i a_{2}, \quad b=b_{1}+i b_{2}, \quad \omega=\omega_{1}+i \omega_{2}, \quad \theta=\theta_{1}+i \theta_{2}$,

then

$$
A(x, y, t)=\sqrt{2}\left[1-\frac{2 i\left(a_{2} x+b_{2} y+\omega_{2} t+\theta_{2}\right)+1}{W}\right],
$$

where

$W=\left(a_{1} x+b_{1} y+\omega_{1} t+\theta_{1}\right)^{2}+\left(a_{2} x+b_{2} y+\omega_{2} t+\theta_{2}\right)^{2}+\Delta$.

The solution $Q$ can also be written down from 2.3 and the above $f$.

This simplest rational solution has three distinctly different dynamical behaviors depending on the parameter value of $p_{1}^{2}$.

1. If $p_{1}^{2}$ is not real, then it is easy to see that $b / a$ is not real, hence $b_{1} / b_{2} \neq a_{1} / a_{2}$. In this case, along the $[x(t), y(t)]$ trajectory where

$$
a_{1} x+b_{1} y=-\omega_{1} t, \quad a_{2} x+b_{2} y=-\omega_{2} t,
$$

$(A, Q)$ are constants. In addition, at any given time, $(A, Q) \rightarrow(\sqrt{2}, 1)$ when $(x, y)$ goes to infinity. Thus the solution is a localized soliton moving on a constant background.

2. If $p_{1}^{2}<0$, i.e., $p_{1}$ is purely imaginary, then $a, b$ and $\omega$ are all imaginary. In this case, the solution is a function of $a_{2} x+b_{2} y+\omega_{2} t$ only, and is thus a line soliton moving on a constant background.

3. If $p_{1}^{2}>0$, i.e., $p_{1}$ is real, then $a, b$ are real but $\omega$ is imaginary. In this case, the solution is also a line wave, but it is not a moving line soliton anymore. As $t \rightarrow \pm \infty$, this line wave goes to a uniform constant background; in the intermediate times, it rises to a higher amplitude. Thus this is a line wave which "appears from nowhere and disappears with no trace", hence it is a line rogue wave.
From the above analysis, we see that rational solutions 2.4 to the DSI equation become rogue waves when the parameters $p_{i}$ are real-valued (this fact holds for $N=1$ as well as for higher $N$ integers). In the next section, we will examine these rogue waves in more detail.

It is noted from the above explicit solution formulae that the parameter $c_{11}$ causes a shift of the origin in the $(x, y, t)$ space. Thus $c_{11}$ can be set to zero by a shift of the $(x, y, t)$ axes. This fact also holds for $N=1$ as well as for higher $N$ integers in the solution (2.4).

\section{ROGUE WAVES IN THE DAVEY-STEWARTSON-I EQUATION}

As we have shown above, rational solutions 2.4 in Theorem 1 become rogue waves in the DSI equation when all parameters $p_{i}$ are required to be real. In this section, we analyze the dynamics of these rogue waves in detail.

\section{A. Fundamental rogue waves}

The fundamental rogue waves in the DSI equation are obtained when one takes $N=1, n_{1}=1$ and $p_{1}$ real in the rational solution 2.4 , and $c_{11}$ is a free complex parameter. After a shift of time and space coordinates, $c_{11}$ can be eliminated and the fundamental rogue waves can be written as

$$
\begin{aligned}
& A(x, y, t)=\sqrt{2}\left[1+\frac{8 i \Omega t-4}{1+\left(k_{1} x+k_{2} y\right)^{2}+4 \Omega^{2} t^{2}}\right], \\
& Q(x, y, t)=1-4 \epsilon k_{1}^{2} \frac{1-\left(k_{1} x+k_{2} y\right)^{2}+4 \Omega^{2} t^{2}}{\left[1+\left(k_{1} x+k_{2} y\right)^{2}+4 \Omega^{2} t^{2}\right]^{2}},
\end{aligned}
$$

where

$$
k_{1}=p_{1}-\epsilon p_{1}^{-1}, \quad k_{2}=p_{1}+\epsilon p_{1}^{-1}, \quad \Omega=p_{1}^{2}+p_{1}^{-2} .
$$

This solution describes a line rogue wave with the line oriented in the $\left(k_{1}, k_{2}\right)$ direction of the $(x, y)$ plane, thus the fundamental rogue waves in the DSI equation are line rogue waves. Along the line direction (with $k_{1} x+k_{2} y$ fixed), the solution is a constant. As $t \rightarrow \pm \infty$, the solution $A$ uniformly approaches the constant background $\sqrt{2}$ everywhere in the $(x, y)$ plane; but in the intermediate times, $|A|$ reaches maximum amplitude $3 \sqrt{2}$ (i.e., three times the background amplitude) on the line $k_{1} x+k_{2} y=0$ at time $t=0$. This fundamental rogue wave is illustrated in Fig. 1 with parameters $\epsilon=1$ and $p_{1}=1.5$.

The above fundamental rogue waves in the DSI equation are two-dimensional counterparts of the fundamental (Peregrine) rogue waves in the NLS equation [4, 5]. Indeed, when we take $\epsilon=1$ and $p_{1}=1$ in the above fundamental rogue waves, we have $k_{1}=0$, hence the solution $A$ is independent of $x$ and $Q=1$. In this case, 

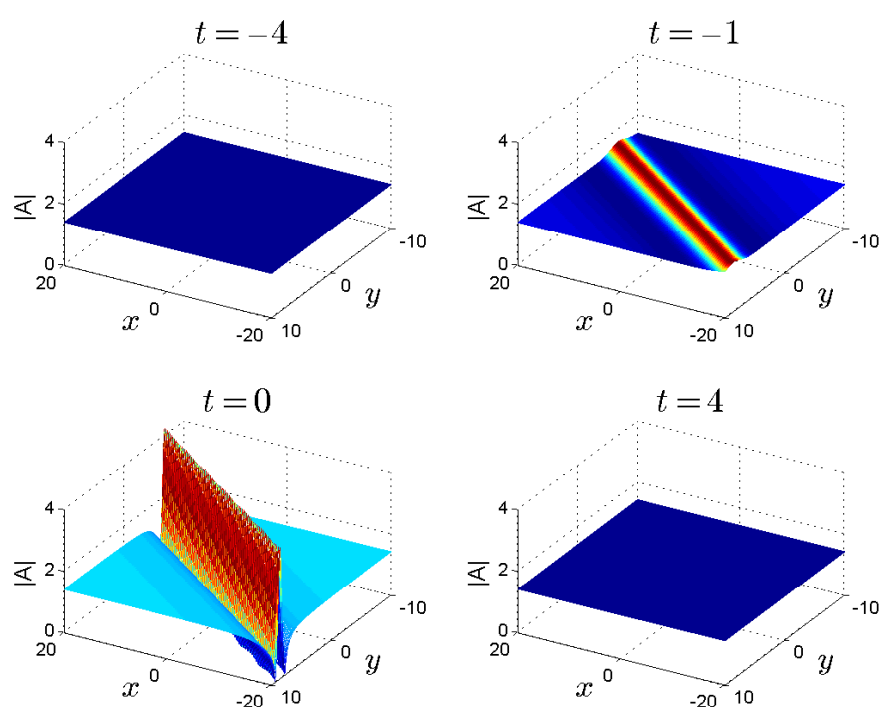

FIG. 1: A fundamental rogue wave 3.1 in the DSI equation with parameters $\epsilon=1, p_{1}=1.5$.

the DSI equation reduces to the NLS equation, and this fundamental rogue wave of the DSI equation reduces to the Peregrine rogue wave of the NLS equation.

Non-fundamental rogue waves can be obtained from the $N$-rational solutions of order $\left(n_{1}, n_{2}, \cdots, n_{N}\right)$ in Eq. (2.4) with real values of $\left(p_{1}, \ldots, p_{N}\right)$ when $N>1$, or $n_{1}>1$, or both. Below we consider two subclasses of these non-fundamental rogue waves.

\section{B. Multi-rogue waves}

One subclass of non-fundamental rogue waves is the multi-rogue waves, which are obtained when we take $N>$ $1, n_{1}=\cdots=n_{N}=1$ in the rational solution (2.4) with real values of $\left(p_{1}, \ldots, p_{N}\right)$. These rogue waves describe the interaction of $N$ individual fundamental rogue waves. When $t \rightarrow \pm \infty$, the solution approaches the constant background uniformly in the entire $(x, y)$ plane. In the intermediate times, $N$ line rogue waves arise from the constant background, interact with each other, and then disappear into the background again. In the far field of the $(x, y)$ plane, the solution consists of $N$ separate line rogue waves. However, in the near field where these line rogue waves intersect and interact, wavefronts of the solution are no longer lines, and interesting curvy wave patterns would appear.

To demonstrate these multi-rogue-wave solutions, we first consider the $N=2$ case. In this case, the $f$ and $g$ functions of the solutions can be obtained from 2.4 as

$$
f=\left|\begin{array}{ll}
m_{11}^{0} & m_{12}^{0} \\
m_{21}^{0} & m_{22}^{0}
\end{array}\right|, \quad g=\left|\begin{array}{ll}
m_{11}^{1} & m_{12}^{1} \\
m_{21}^{1} & m_{22}^{1}
\end{array}\right|
$$

where

$$
\begin{aligned}
m_{i j}^{0}=\frac{1}{p_{i}+\bar{p}_{j}}[ & \left(\xi_{i}^{\prime}+c_{i 1}-\frac{p_{i}}{p_{i}+\bar{p}_{j}}\right) \times \\
& \left.\left(\bar{\xi}_{j}^{\prime}+\bar{c}_{j 1}-\frac{\bar{p}_{j}}{p_{i}+\bar{p}_{j}}\right)+\frac{p_{i} \bar{p}_{j}}{\left(p_{i}+\bar{p}_{j}\right)^{2}}\right], \\
m_{i j}^{1}=\frac{1}{p_{i}+\bar{p}_{j}}[ & \left(\xi_{i}^{\prime}+1+c_{i 1}-\frac{p_{i}}{p_{i}+\bar{p}_{j}}\right) \times \\
& \left.\left(\bar{\xi}_{j}^{\prime}-1+\bar{c}_{j 1}-\frac{\bar{p}_{j}}{p_{i}+\bar{p}_{j}}\right)+\frac{p_{i} \bar{p}_{j}}{\left(p_{i}+\bar{p}_{j}\right)^{2}}\right],
\end{aligned}
$$

$\xi_{j}^{\prime}$ is given by Eq. 2.6), $p_{1}, p_{2}$ are free real parameters, and $c_{11}, c_{21}$ are tree complex parameters. The complex parameter $c_{11}$ can be removed by a shift of the $(x, y, t)$ axes, then this two-rogue-wave solution contains four non-trivial real parameters, namely, $p_{1}, p_{2}$, and the real and imaginary parts of $c_{21}$. This solution for parameters

$$
\epsilon=1, p_{1}=1, p_{2}=1.5, c_{11}=c_{21}=0
$$

is shown in Fig. 2, It is seen that when these two line rogue waves arise from the constant background, the region of their intersection acquires higher amplitude first (see $t=-1$ panel). After these higher amplitudes in the intersection region fade, the line-rogue solutions in the far field then rise to higher amplitude (see $t=0$ panel). Interestingly, the wave pattern at $t=0$ features two curvy wavefronts which are well separated. These curvy wavefronts are caused by the interaction of the two fundamental (line) rogue waves. At large times, the solution goes back to the constant background again (see $t=5$ panel).

For larger $N$, these multi-rogue waves have qualitatively similar behaviors, except that more line-rogue waves will arise and interact with each other, and more complicated wavefronts will form in the interaction region. For example, with $N=3$ and parameter choices

$$
\epsilon=1, p_{1}=1, p_{2}=1.5, p_{3}=2, c_{11}=c_{21}=c_{31}=0,
$$

the corresponding solution is shown in Fig. 3. As can be seen, the transient solution patterns become more intricate.

\section{Higher-order rogue waves}

Another subclass of non-fundamental rogue waves is the higher-order rogue waves, which are obtained when we take $N=1$ and $n_{1}>1$ in the rational solution 2.4 with a real value of $p_{1}$. For instance, if $n_{1}=2$, we get second-order rogue waves from 2.4 as

$$
\begin{aligned}
f= & {\left[\left(p_{1} \partial_{p_{1}}+\xi_{1}^{\prime}\right)^{2}+c_{12}\right] } \\
& {\left[\left(\bar{p}_{1} \partial_{\bar{p}_{1}}+\bar{\xi}_{1}^{\prime}\right)^{2}+\bar{c}_{12}\right] \frac{1}{p_{1}+\bar{p}_{1}}, }
\end{aligned}
$$



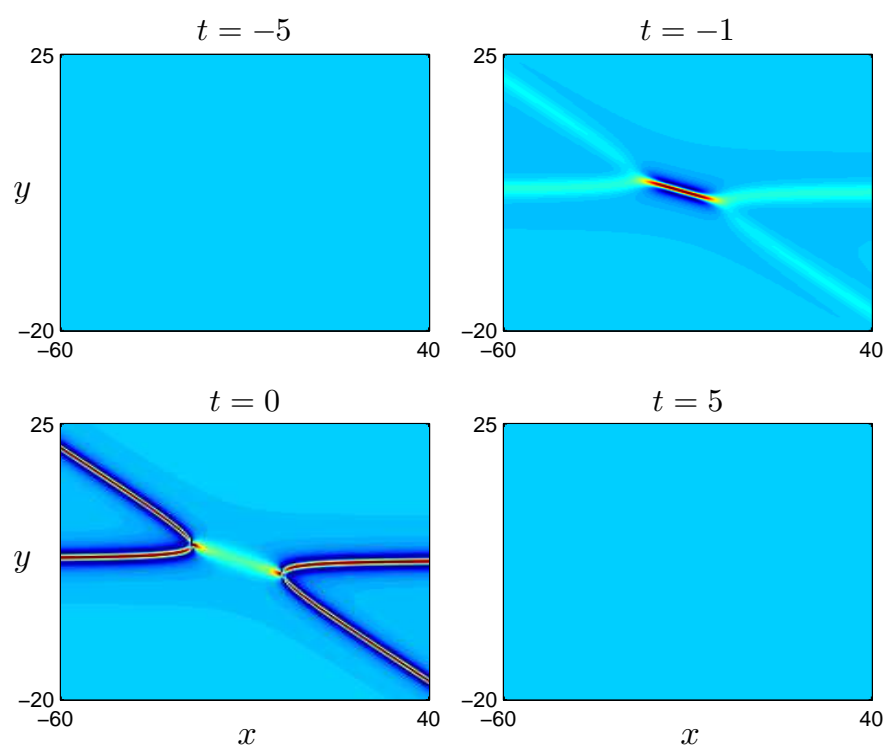

FIG. 2: A two-rogue-wave solution 3.3 in the DSI equation for parameters (3.4). Plotted is the $|A|$ field. The constantbackground value is $\sqrt{2}$.

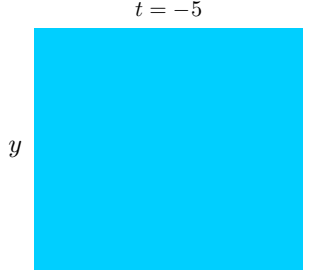

$t=0$
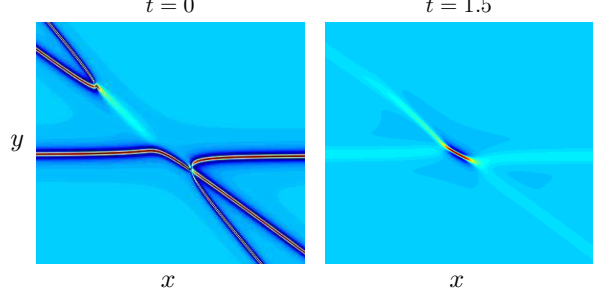

FIG. 3: A three-rogue-wave solution in the DSI equation for parameters 3.5. Plotted is the $|A|$ field.

$$
\begin{aligned}
g= & {\left[\left(p_{1} \partial_{p_{1}}+\xi_{1}^{\prime}+1\right)^{2}+c_{12}\right] } \\
& {\left[\left(\bar{p}_{1} \partial_{\bar{p}_{1}}+\bar{\xi}_{1}^{\prime}-1\right)^{2}+\bar{c}_{12}\right] \frac{1}{p_{1}+\bar{p}_{1}}, }
\end{aligned}
$$

where $\xi_{1}^{\prime}$ is given by Eq. 2.6), $p_{1}$ is a free real parameter, and $c_{12}$ is a free complex parameter. Here we have set $c_{11}=0$ in 2.4 by a shift of the $(x, y, t)$ axes. Higherorder rogue waves with $n_{1}>2$ can be similarly obtained.

An interesting phenomenon is that, unlike the multirogue waves discussed in the previous subsection, these higher-order rogue waves do not uniformly approach the constant background as $t \rightarrow \pm \infty$. Instead, only parts of their wave structures approach the constant background as $t \rightarrow \pm \infty$, but the other parts move to the far distance as localized lumps with undiminished amplitude and in- creasing velocity as $t \rightarrow \pm \infty$. To illustrate these behaviors, we consider the above second-order rogue waves. For parameter values

$$
\epsilon=1, \quad p_{1}=1, \quad c_{12}=0,
$$

we get

$$
\begin{gathered}
f=\frac{1}{2}\left[x-\left(4 t^{2}+y-y^{2}\right)\right]^{2}+\left[\left(y-\frac{1}{2}\right)^{2}+\frac{1}{4}\right]\left(8 t^{2}+\frac{1}{2}\right), \\
g=f-4 i t\left(x+y-y^{2}-4 t^{2}\right)+x+y-y^{2}-12 t^{2}-\frac{1}{2},
\end{gathered}
$$

and

$$
A(x, y, t)=\sqrt{2} \frac{g}{f} .
$$

For this solution, $\bar{A}(x, y, t)=A(x, y,-t)$, thus $|A(x, y,-t)|=|A(x, y, t)|$. This solution is displayed in Fig. 4. We see that when $|t| \gg 1$, the solution is a localized lump sitting on the constant background $\sqrt{2}$ (see $t= \pm 7$ panels). The peak amplitude of the lump is attained at

$$
(x, y)=\left(4 t^{2}+\frac{1}{4}, \frac{1}{2}\right),
$$

thus this lump is accelerating rightward as $|t|$ increases (see $t=-7$ and $t=-5$ panels). The peak-amplitude value of the lump stays at $3 \sqrt{2}$ and is unchanged though. As the lump accelerates rightward (with increasing $|t|$ ), its vertical size (along the $y$ direction) remains the same, but its horizontal size (along the $x$ direction) expands (see $t=-7,-5,-1$ panels). When $t \rightarrow 0$, this lump disappears. At the same time, a parabola-shaped rogue wave rises from the background (see $t=-1$ and $t=0$ panels). At $t=0$, this parabola is located at

$$
x=y-y^{2},
$$

where the rogue wave reaches peak amplitude $3 \sqrt{2}$ (see $t=0$ panel). Visually one may describe the solution in Fig. 4 as an incoming lump being reflected back by the emergence of a parabola-shaped rogue wave. In addition, this lump decelerates as it comes afar and accelerates as it goes away.

We have also examined the second-order rogue waves (3.6)-3.7) for other parameter choices of $\left(\epsilon, p_{1}, c_{12}\right)$, and found that those solutions are qualitatively the same as the one in Fig. 4, except that those solution patterns may be stretched and skewed in the $(x, y)$ plane.

\section{SUMMARY}

In summary, we have derived general rogue waves in the DSI equation by the bilinear method, and our solutions are given in terms of determinants. We showed that 


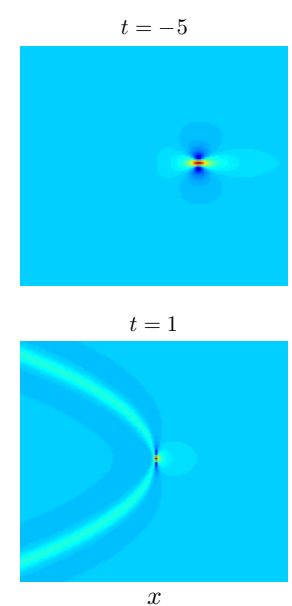



$t=7$

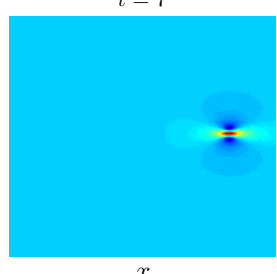

$x$
FIG. 4: A second-order rogue wave solution 3.9 in the DSI equation with $\epsilon=1$.

the simplest (fundamental) rogue waves are line rogue waves which arise from the constant background with a line profile and then disappear into the constant background again (see Fig. 1). We also showed that multirogue waves describe the interaction of several fundamental rogue waves, and interesting curvy wave patterns appear due to this interaction (see Figs. 2 and 3). However, higher-order rogue waves were found to show very novel features. Specifically, only parts of the wave structures in the higher-order rogue waves rise from the constant background and then retreat back to it, but the other parts of the waves come from the far distance as localized lumps, which interact with the transient rogue waves in the near field and then are reflected back and accelerate to the large distance again (see Fig. 4). These rogue wave solutions to the DSI equation generalize the rogue waves of the NLS equation into two spatial dimensions, and they could play a role in the physical understanding of rogue water waves in the ocean.

\section{Acknowledgment}

The work of Y.O. is supported in part by JSPS Grantin-Aid for Scientific Research (B-19340031, S-19104002) and for challenging Exploratory Research (22656026), and the work of J.Y. is supported in part by the Air Force Office of Scientific Research (Grant USAF 955009-1-0228) and the National Science Foundation (Grant DMS-0908167).

\section{Appendix}

In this appendix we will prove Theorem 1 in section II by using the bilinear method. Firstly we present the following lemma.
Lemma 1 Let $m_{i j}^{(n)}, \varphi_{i}^{(n)}$ and $\psi_{j}^{(n)}$ be functions of $x_{1}$, $x_{2}, x_{-1}$ and $x_{-2}$ satisfying the following differential and difference relations,

$$
\begin{aligned}
& \partial_{x_{1}} m_{i j}^{(n)}=\varphi_{i}^{(n)} \psi_{j}^{(n)} \\
& \partial_{x_{2}} m_{i j}^{(n)}=\varphi_{i}^{(n+1)} \psi_{j}^{(n)}+\varphi_{i}^{(n)} \psi_{j}^{(n-1)}, \\
& \partial_{x_{-1}} m_{i j}^{(n)}=-\varphi_{i}^{(n-1)} \psi_{j}^{(n+1)} \\
& \partial_{x_{-2}} m_{i j}^{(n)}=-\varphi_{i}^{(n-2)} \psi_{j}^{(n+1)}-\varphi_{i}^{(n-1)} \psi_{j}^{(n+2)}, \\
& m_{i j}^{(n+1)}=m_{i j}^{(n)}+\varphi_{i}^{(n)} \psi_{j}^{(n+1)} \\
& \partial_{x_{\nu}} \varphi_{i}^{(n)}=\varphi_{i}^{(n+\nu)}, \\
& \partial_{x_{\nu}} \psi_{j}^{(n)}=-\psi_{j}^{(n-\nu)}, \quad(\nu=1,2,-1,-2) .
\end{aligned}
$$

Then the determinant,

$$
\tau_{n}=\operatorname{det}_{1 \leq i, j \leq N}\left(m_{i j}^{(n)}\right),
$$

satisfies the bilinear equations,

$$
\begin{aligned}
& \left(D_{x_{1}} D_{x_{-1}}-2\right) \tau_{n} \cdot \tau_{n}=-2 \tau_{n+1} \tau_{n-1} \\
& \left(D_{x_{1}}^{2}-D_{x_{2}}\right) \tau_{n+1} \cdot \tau_{n}=0 \\
& \left(D_{x_{-1}}^{2}+D_{x_{-2}}\right) \tau_{n+1} \cdot \tau_{n}=0
\end{aligned}
$$

This lemma can be proved by the same method as for Lemma 3.1 in [12], thus its proof is omitted here. We note that by the variable transformation

$x_{1}=\frac{1}{2}(x+y), \quad x_{-1}=\frac{\epsilon}{2}(x-y), \quad x_{2}=-\frac{i}{2} t, \quad x_{-2}=\frac{i}{2} t$

and the complex conjugate condition

$$
\bar{\tau}_{n}=\tau_{-n},
$$

the above bilinear equations A.3 are reduced to the bilinear form 2.2 of the DSI equation for $f=\tau_{0}$ and $g=\tau_{1}$. Therefore all we need is to choose appropriate matrix elements $m_{i j}^{(n)}$ which satisfy A.1) and realize the conjugate condition A.5 with A.4.

Proof of Theorem 1 It is easy to see that functions $\varphi_{i}^{(n)}, \psi_{j}^{(n)}$ and $m_{i j}^{(n)}$ defined by

$$
\begin{gathered}
\varphi_{i}^{(n)}=p_{i}^{n} e^{\xi_{i}}, \quad \psi_{j}^{(n)}=\left(-q_{j}\right)^{-n} e^{\eta_{j}} \\
m_{i j}^{(n)}=\int^{x_{1}} \varphi_{i}^{(n)} \psi_{j}^{(n)} d x_{1}=\frac{1}{p_{i}+q_{j}}\left(-\frac{p_{i}}{q_{j}}\right)^{n} e^{\xi_{i}+\eta_{j}}, \\
\xi_{i}=\frac{1}{p_{i}^{2}} x_{-2}+\frac{1}{p_{i}} x_{-1}+p_{i} x_{1}+p_{i}^{2} x_{2}, \\
\eta_{j}=-\frac{1}{q_{j}^{2}} x_{-2}+\frac{1}{q_{j}} x_{-1}+q_{j} x_{1}-q_{j}^{2} x_{2},
\end{gathered}
$$


satisfy Eqs. A.1. Here $p_{i}, q_{j}$ are arbitrary complex constants, and it is assumed that the lower boundary value of the integral in the above $m_{i j}^{(n)}$ equation is zero. But these functions do not lead to rational solutions.

To get rational solutions, we differentiate the above functions with respect to the parameters $p_{i}$ and $q_{j}$. To obtain solution expressions in (a) of Theorem 1, we consider the following $\varphi_{i}^{(n)}, \psi_{j}^{(n)}$ and $m_{i j}^{(n)}$ functions:

$$
\begin{array}{r}
\varphi_{i}^{(n)}=A_{i} p_{i}^{n} e^{\xi_{i}}, \quad \psi_{j}^{(n)}=B_{j}\left(-q_{j}\right)^{-n} e^{\eta_{j}}, \\
m_{i j}^{(n)}=A_{i} B_{j} \frac{1}{p_{i}+q_{j}}\left(-\frac{p_{i}}{q_{j}}\right)^{n} e^{\xi_{i}+\eta_{j}},
\end{array}
$$

where $A_{i}$ and $B_{j}$ are differential operators of order $n_{i}$ and $n_{j}$ with respect to $p_{i}$ and $q_{j}$ respectively, defined as

$$
A_{i}=\sum_{k=0}^{n_{i}} c_{i k}\left(p_{i} \partial_{p_{i}}\right)^{n_{i}-k}, \quad B_{j}=\sum_{l=0}^{n_{j}} d_{j l}\left(q_{j} \partial_{q_{j}}\right)^{n_{j}-l},
$$

$c_{i k}, d_{j l}$ are arbitrary complex constants, and $n_{i}$ are arbitrary positive integers. It is easy to see that these functions also satisfy Eqs. A.1, thus $\tau_{n}=\operatorname{det}\left(m_{i j}^{(n)}\right)$ with A.7) satisfies the bilinear equations A.3. By using the operator relations

$$
\begin{aligned}
\left(p_{i} \partial_{p_{i}}\right) p_{i}^{n} e^{\xi_{i}} & =p_{i}^{n} e^{\xi_{i}}\left(p_{i} \partial_{p_{i}}+\xi_{i}^{\prime}+n\right), \\
\left(q_{j} \partial_{q_{j}}\right)\left(-q_{j}\right)^{-n} e^{\eta_{j}} & =\left(-q_{j}\right)^{-n} e^{\eta_{j}}\left(q_{j} \partial_{q_{j}}+\eta_{j}^{\prime}-n\right),
\end{aligned}
$$

where

$$
\begin{gathered}
\xi_{i}^{\prime}=-\frac{2}{p_{i}^{2}} x_{-2}-\frac{1}{p_{i}} x_{-1}+p_{i} x_{1}+2 p_{i}^{2} x_{2}, \\
\eta_{j}^{\prime}=\frac{2}{q_{j}^{2}} x_{-2}-\frac{1}{q_{j}} x_{-1}+q_{j} x_{1}-2 q_{j}^{2} x_{2},
\end{gathered}
$$

the matrix element $m_{i j}^{(n)}$ in A.7 becomes

$$
\begin{aligned}
m_{i j}^{(n)}= & \left(-\frac{p_{i}}{q_{j}}\right)^{n} e^{\xi_{i}+\eta_{j}} \sum_{k=0}^{n_{i}} c_{i k}\left(p_{i} \partial_{p_{i}}+\xi_{i}^{\prime}+n\right)^{n_{i}-k} \\
& \sum_{l=0}^{n_{j}} d_{j l}\left(q_{j} \partial_{q_{j}}+\eta_{j}^{\prime}-n\right)^{n_{j}-l} \frac{1}{p_{i}+q_{j}} .
\end{aligned}
$$

Taking parameter constraints

$$
q_{j}=\bar{p}_{j}, \quad d_{j l}=\bar{c}_{j l},
$$

and using the variable transformation (A.4), we obtain

$$
\eta_{j}=\bar{\xi}_{j}, \quad \overline{m_{i j}^{(n)}}=m_{j i}^{(-n)}, \quad \bar{\tau}_{n}=\tau_{-n},
$$

thus the conjugate condition A.5 is satisfied. Finally using the gauge freedom of $\tau_{n}$, we obtain the rational solutions to the DSI equation as given in (a) of Theorem 1.
Next we derive solution expressions in (b) of Theorem 1. For this purpose, we consider the following $\varphi_{i}^{(n)}, \psi_{j}^{(n)}$ and $m_{i j}^{(n)}$ functions:

$$
\begin{gathered}
\varphi_{i}^{(n)}=\hat{A}_{i} p_{i}^{n} e^{\xi_{i}}, \quad \psi_{j}^{(n)}=\hat{B}_{j}\left(-q_{j}\right)^{-n} e^{\eta_{j}}, \\
m_{i j}^{(n)}=\int^{x_{1}} \varphi_{i}^{(n)} \psi_{j}^{(n)} d x_{1},
\end{gathered}
$$

where $\hat{A}_{i}$ and $\hat{B}_{j}$ are differential operators of order $n_{i}$ and $n_{j}$ with respect to $p_{i}$ and $q_{j}$ defined as

$$
\begin{aligned}
& \hat{A}_{i}=\sum_{k=0}^{n_{i}} \frac{\hat{c}_{i k}}{\left(n_{i}-k\right) !}\left(p_{i} \partial_{p_{i}}\right)^{n_{i}-k}, \\
& \hat{B}_{j}=\sum_{l=0}^{n_{j}} \frac{\hat{d}_{j l}}{\left(n_{j}-l\right) !}\left(q_{j} \partial_{q_{j}}\right)^{n_{j}-l},
\end{aligned}
$$

$\hat{c}_{i k}, \hat{d}_{j l}$ are arbitrary complex constants which are related to the constants $c_{i k}, d_{j l}$ in the solution expression (a) as

$$
\hat{c}_{i k}=c_{i k}\left(n_{i}-k\right) !, \quad \hat{d}_{j l}=d_{j l}\left(n_{j}-l\right) !,
$$

and it is assumed that the lower boundary value of the integral in A.11 is zero. Rewriting $\varphi_{i}^{(n)}$ and $\psi_{j}^{(n)}$ in A.10 as

$$
\begin{aligned}
\hat{A}_{i} p_{i}^{n} e^{\xi_{i}} & =P_{i}^{(n)} p_{i}^{n} e^{\xi_{i}}, \\
\hat{B}_{j}\left(-q_{j}\right)^{-n} e^{\eta_{j}} & =Q_{j}^{(n)}\left(-q_{j}\right)^{-n} e^{\eta_{j}},
\end{aligned}
$$

where $P_{i}^{(n)}$ and $Q_{j}^{(n)}$ are polynomials of degrees $n_{i}$ and $n_{j}$ in $\left(x_{-2}, x_{-1}, x_{1}, x_{2}\right)$ respectively, and using the integration by parts, $m_{i j}^{(n)}$ in A.11 then turns into

$$
m_{i j}^{(n)}=\left(-\frac{p_{i}}{q_{j}}\right)^{n} e^{\xi_{i}+\eta_{j}} \sum_{\nu=0}^{n_{i}+n_{j}} \frac{(-1)^{\nu}}{\left(p_{i}+q_{j}\right)^{\nu+1}} \partial_{x_{1}}^{\nu} P_{i}^{(n)} Q_{j}^{(n)} .
$$

Due to the gauge freedom of $\tau_{n}$, we can see that

$$
m_{i j}^{(n)}=\sum_{\nu=0}^{n_{i}+n_{j}}\left(\frac{-1}{p_{i}+q_{j}}\right)^{\nu+1} \partial_{x_{1}}^{\nu} P_{i}^{(n)} Q_{j}^{(n)}
$$

gives the same solution. In order for this solution to satisfy the conjugate condition A.5, we take

$$
q_{j}=\bar{p}_{j}, \quad \hat{d}_{j l}=\overline{\hat{c}}_{j l} .
$$

Then in view of the variable transformation A.4, we have

$$
\overline{Q_{j}^{(n)}}=P_{j}^{(-n)}, \quad \overline{m_{i j}^{(n)}}=m_{j i}^{(-n)}, \quad \bar{\tau}_{n}=\tau_{-n},
$$

hence $\tau_{n}$ with the above matrix elements A.16) satisfies the bilinear DSI equation (2.2). 
Lastly we derive the explicit expression of $P_{i}^{(n)}$. From the definition of $P_{i}^{(n)}$ in A.14, we have

$$
\sum_{k=0}^{n_{i}} \frac{\hat{c}_{i k}}{\left(n_{i}-k\right) !}\left(p_{i} \partial_{p_{i}}\right)^{n_{i}-k} p_{i}^{n} e^{\xi_{i}}=P_{i}^{(n)} p_{i}^{n} e^{\xi_{i}}
$$

For $\xi=\sum_{\nu=-2,-1,1,2} p^{\nu} x_{\nu}$, using the functional identity (see Appendix of [12])

$$
e^{\kappa p \partial_{p}} F(p)=F\left(e^{\kappa} p\right)
$$

we get

$$
\begin{aligned}
\frac{1}{p^{n} e^{\xi}} e^{\kappa p \partial_{p}} p^{n} e^{\xi} & =e^{\kappa n} \exp \left(\sum_{\nu}\left(e^{\nu \kappa}-1\right) p^{\nu} x_{\nu}\right) \\
& =\exp \left(\kappa n+\sum_{k=1}^{\infty} \frac{\kappa^{k}}{k !} \sum_{\nu} \nu^{k} p^{\nu} x_{\nu}\right) \\
& =\sum_{k=0}^{\infty} \kappa^{k} S_{k}\left(\boldsymbol{\xi}^{(n)}(p)\right),
\end{aligned}
$$

where $\boldsymbol{\xi}^{(n)}(p)=\left(\xi_{1}(p)+n, \xi_{2}(p), \cdots, \xi_{k}(p)+\delta_{k 1} n, \cdots\right)$ and $\xi_{k}(p)=\sum_{\nu} \nu^{k} p^{\nu} x_{\nu} / k$ !. By comparing the coefficient of order $\kappa^{k}$ in Eq. A.20, we obtain

$$
\frac{1}{p^{n} e^{\xi}} \frac{\left(p \partial_{p}\right)^{k}}{k !} p^{n} e^{\xi}=S_{k}\left(\boldsymbol{\xi}^{(n)}(p)\right)
$$

Substituting $p=p_{i}$ and $\xi=\xi_{i}$ into this equation and inserting it into A.19, and recalling the variable transformation A.4, we then find the explicit expression for $P_{i}^{(n)}$ as

$$
P_{i}^{(n)}=\sum_{k=0}^{n_{i}} \hat{c}_{i k} S_{n_{i}-k}\left(\boldsymbol{\xi}^{(n)}\left(p_{i}\right)\right)
$$

where $\boldsymbol{\xi}^{(n)}\left(p_{i}\right)$ is as given in Theorem 1. Inserting this $P_{i}^{(n)}$ and $Q_{j}^{(n)}$ from A.18 into A.16, the solution expression in (b) of Theorem 1 is then derived. This ends the proof of Theorem 1.
[1] C. Kharif, E. Pelinovsky and A. Slunyaev, Rogue waves in the ocean (Springer, Berlin, 2009).

[2] D. R. Solli, C. Ropers, P. Koonath and B. Jalali, "Optical rogue waves", Nature 450, 1054-1057 (2007).

[3] B. Kibler, J. Fatome, C. Finot, G. Millot, F. Dias, G. Genty, N. Akhmediev, J.M. Dudley, "The Peregrine soliton in nonlinear fibre optics", Nature Physics, 6, 790-795 (2010).

[4] D. H. Peregrine, "Water waves, nonlinear Schrödinger equations and their solutions," J. Australian Math. Soc. B, 25, 16-43 (1983).

[5] N. Akhmediev, A. Ankiewicz, and J. M. Soto-Crespo, "Rogue Waves and Rational Solutions of the Nonlinear Schrdinger Equation," Phys. Rev. E 80, 026601 (2009).

[6] P. Dubard, P. Gaillard, C. Klein, V.B. Matveev, "On multi-rogue wave solutions of the NLS equation and positon solutions of the KdV equation", Eur. Phys. J. Special Topics 185, 247-258 (2010).

[7] P. Dubard, V.B. Matveev, "Multi-rogue waves solutions to the focusing NLS equation and the KP-I equation", Nat. Hazards. Earth. Syst. Sci. 11, 667-672 (2011).

[8] P. Gaillard, "Families of quasi-rational solutions of the NLS equation and multi-rogue waves", J. Phys. A: Math. Theor. 44, 435204 (2011).

[9] A. Ankiewicz, D.J. Kedziora and N. Akhmediev, "Rogue wave triplets", Phys. Lett. A, 375, 2782-2785 (2011).

[10] D.J. Kedziora, A. Ankiewicz, and N. Akhmediev, "Circular rogue wave clusters", Phys. Rev. E 84, 056611 (2011).

[11] B. Guo, L. Ling, and Q.P. Liu, "Nonlinear Schrödinger equation: Generalized Darboux transformation and rogue wave solutions", Phys. Rev. E 85, 026607 (2012).

[12] Y. Ohta and J. Yang, "General high-order rogue waves and their dynamics in the nonlinear Schrödinger equa- tion", Proc. Roy. Soc. A. 468, 1716-1740 (2012).

[13] A. Ankiewicz, J. M. Soto-Crespo, and N. Akhmediev, "Rogue waves and rational solutions of the Hirota equation", Phys. Rev. E, 81, 046602 (2010).

[14] N. Akhmediev, V.M. Eleonskii, and N.E. Kulagin, "Generation of a periodic sequence of picosecond pulses in an optical fiber: Exact solutions", Sov. Phys. JETP, 62, 894 (1985).

[15] N. Akhmediev, V.M. Eleonskii and N.E. Kulagin, "Exact first-order solutions of the nonlinear Schödinger equation", Theor. Math. Phys. 72, 809-818 (1988).

[16] A.R. Its, A.V. Rybin, and M.A. Salle, "Exact Integration of Nonlinear Schrödinger equation", Theor. Math. Phys. 74, 29-45 (1988).

[17] M.J. Ablowitz and B.M Herbst, "On homoclinic structure and numerically induced chaos for the nonlinear Schr6dinger equation", SIAM J. Appl. Math. 50, 339 (1990).

[18] N. Akhmediev, A. Ankiewicz and M. Taki, "Waves that appear from nowhere and disappear without a trace", Phys. Lett. A 373, 675-678 (2009).

[19] D. J. Benney and G. Roskes, "Wave instabilities", Stud. Appl. Math. 48, 377-385 (1969).

[20] A. Davey and K. Stewartson, "On three-dimensional packets of surface waves", Proc. R. Soc. London, A 338, 101-110 (1974).

[21] M.J. Ablowitz and H. Segur, Solitons and the Inverse Scattering Transform (SIAM, Philadelphia, 1981).

[22] M. Tajiri and T. Arai, "Growing-and-decaying mode solution to the Davey-Stewartson equation", Phys. Rev. E 60, 2297 (1999). 